\title{
The Study of the Heisenberg-Euler Lagrangian and Some of its Applications
}

\author{
S. R. Valluri \\ Departments of Physics, Astronomy \& Applied Mathematics, \\ University of Western Ontario, London, ON, N6A 3K7, Canada \\ valluri@uwo.ca \\ U. D. Jentschura \\ National Institute of Standards and Technology, \\ Mail Stop 8401, Gaithersburg, Maryland, 20899-8401, USA \\ ulj@nist.gov \\ D. R. Lamm \\ Electro-Optics, Environment, and Materials Laboratory, \\ GTRI, Georgia Institute of Technology, Atlanta, GA, 30332, USA \\ darrell.lamm@gtri.gatech.edu
}

\begin{abstract}
The Heisenberg-Euler Lagrangian (HEL) is not only a topic of fundamental interest, but also has a rich variety of diverse applications in astrophysics, nonlinear optics and elementary particle physics etc. We discuss the series representation of this Lagrangian and a few of its applications in this study. [In an appendix, we discuss issues related to the renormalization - and the renormalization-group invariance- of the HEL and its two-loop generalization.]
\end{abstract}




\section{INTRODUCTION}

The effective one-loop Lagrangian density [1-11] of quantum electrodynamics (QED) describes the nonlinear interaction of electromagnetic fields due to a single closed electron loop. [It is also referred to as the QED effective action, or the effective Lagrangian.] One of the most compact and elegant ways to treat the symmetry properties of the vacuum is by the method of the effective Lagrangian [12].

This one-loop Lagrangian, often called the Heisenberg-Euler Lagrangian (HEL), has been used to describe a variety of electromagnetic processes. The real part of the Lagrangian can be used to delineate such dispersive phenomena as photon propagation in a magnetic field, second harmonic generation, photon splitting in a magnetic field, and light scattering in a vacuum [1,4-9,11,13-17]. The development of high intensity lasers, with the consequent availability of powerful, coherent light sources, can render possible the observation of delicate nonlinear effects and also the nonlinearity of Maxwell's equations. Strong magnetic fields around pulsars and around magnetars, and other astrophysical and laboratory situations might also warrant accurate calculations based on the Lagrangian for astrophysical and other applications. The imaginary part of the Lagrangian has been applied to absorption processes such as electron-positron pair creation [4, 5, 8, 9 .

While the integral representation of this Lagrangian as a function of the electric and magnetic field is well known, further analytical representations and numerical procedures for arbitrary field strengths have recently been refined. In the special cases when either the magnetic or electric field vanishes, the Heisenberg-Euler Lagrangian may be expressed in terms of elementary functions and integrals of the natural logarithm of the generalized gamma function with real or complex arguments 18 19. Elementary function series expansions for these gamma function integrals and precise numerical values for the Lagrangian using the expressions of Dittrich et al. were provided by Valluri et al. 18. For the more general case when both an electric and magnetic field are present, a numerically useful, analytical series expression for the real part of the Lagrangian was derived and this latter series expression involves elementary functions and the sine, cosine, and exponential integrals, all of which are easily calculated [20].

Motivations for studying non-linear generalizations of Maxwell's equations in the vacuum are now quite different. Strong interest in one-loop corrections to the classical Lagrangian in Abelian (as well as non-Abelian) gauge theories has resurged in order to learn about the structure of the vacuum when it is probed by an external electromagnetic field. The problem of the existence of a stable electron has been and continues to be an interesting issue, not only relevant to pure electrodynamics but also to an unknown fundamental theory of matter and its interactions (for an interesting view on this issue, see [12]).

In this paper we discuss some analytic calculations relevant for the applications of the HEL. The paper is organized as follows. Section 2 provides the analytical definitions and preliminaries. Section 3 presents the analytical results indicating the existence of the higher harmonics for the real part of the HEL, and the discussion of these results will conclude the main body of the paper. Some aspects of the renormalization and the renormalization-group invariance of the action are briefly discussed in an Appendix. The final section summarizes the conclusions. 


\section{REPRESENTATION OF THE QED EFFECTIVE ACTION BY SPECIAL FUNCTIONS}

The renormalized (see also the Appendix) Heisenberg-Euler Lagrangian (HEL) $\Delta \mathcal{L}$ is expressed as a one-dimensional proper-time integral [1-4]:

$$
\Delta \mathcal{L}=-\frac{e^{2}}{8 \pi^{2}} \lim _{\epsilon, \eta \rightarrow 0^{+}} \int_{\eta}^{i \infty+\eta} \frac{d s}{s} e^{-\left(m^{2}-i \epsilon\right) s}\left[a b \operatorname{coth}(e a s) \cot (e b s)-\frac{a^{2}-b^{2}}{3}-\frac{1}{(e s)^{2}}\right]
$$

and is a quantum correction to the Maxwellian Lagrangian

$$
L_{c l}=L_{0}=L_{M}=\frac{1}{2}\left(E^{2}-B^{2}\right) .
$$

$\Delta \mathcal{L}$ is a one-dimensional proper time integral with proper-time parameter $s$ and can be evaluated by numerical quadrature. A convergent series expansion with (computational and conceptual advantages) can be derived in terms of special functions. There is a unified series expansion encompassing both the real and the imaginary part [20. We need techniques for a reliable numerical evaluation which may have a variety of applications. While the series expansion might be regarded as a complete solution from a theoretical point of view, the expansion alone does not solve the physics associated with the Lagrangian, and it does not provide eo ipso a general, numerically efficient algorithm for its evaluation. The integral defining the HEL, which can be calculated numerically by quadrature, has a slow convergence due to oscillations of the integrand.

The QED effective Lagrangian can be expressed as a function of the Lorentz invariants $\mathcal{F}$ and $\mathcal{G}$, given by

$$
\begin{gathered}
\mathcal{F}=\frac{1}{4} F_{\mu \nu} F^{\mu \nu}=\frac{1}{2}\left(\boldsymbol{B}^{2}-\boldsymbol{E}^{2}\right)=\frac{1}{2}\left(a^{2}-b^{2}\right), \\
\mathcal{G}=\frac{1}{4} F_{\mu \nu}(* F)^{\mu \nu}=-\boldsymbol{E} \cdot \boldsymbol{B}= \pm a b,
\end{gathered}
$$

where $\boldsymbol{E}$ and $\boldsymbol{B}$ are the electric and magnetic field strengths, $F_{\mu \nu}$ is the field-strength tensor and $(* F)^{\mu \nu}$ denotes the dual field-strength $(* F)^{\mu \nu}=(1 / 2) \epsilon^{\mu \nu \rho \sigma} F_{\rho \sigma}$. The quantities $a$ and $b$ denote secular invariants,

$$
\begin{aligned}
a & =\sqrt{\sqrt{\mathcal{F}^{2}+\mathcal{G}^{2}}+\mathcal{F}}, \\
b & =\sqrt{\sqrt{\mathcal{F}^{2}+\mathcal{G}^{2}}-\mathcal{F}} .
\end{aligned}
$$

Secular invariants emerge naturally as eigenvalues of the field-strength tensor fields. We also note that in the case $\mathcal{G}<0$, it is possible to choose a Lorentz frame in which $E$ and $B$ are parallel.

$$
a=|\boldsymbol{B}| \text { and } b=|\boldsymbol{E}| \text { if } \boldsymbol{B} \text { is (anti-)parallel to } \boldsymbol{E},
$$

$a$ and $b$ are positive definite and this gives the condition:

$$
a b=|\boldsymbol{E} \cdot \boldsymbol{B}|>0 \text { for any Lorentz frame and } \mathcal{G} \neq 0 .
$$


Also, the following notation is sometimes used:

$$
\begin{gathered}
\mathcal{F}=-S, \quad \mathcal{G}=-P, \\
\mathcal{L}_{\mathrm{cl}}=-\mathcal{F}=-\frac{1}{4} F_{\mu \nu} F^{\mu \nu}=\frac{1}{2}\left(\boldsymbol{E}^{2}-\boldsymbol{B}^{2}\right)=\frac{1}{2}\left(b^{2}-a^{2}\right) .
\end{gathered}
$$

The correction $\Delta \mathcal{L}$ to the Maxwellian Lagrangian $L_{0}$ can be written in terms of the secular invariants $a$ and $b[8,9$,

$$
\Delta \mathcal{L}=\operatorname{Re} \Delta \mathcal{L}+\mathrm{i} \operatorname{Im} \Delta \mathcal{L}
$$

the real part can be expressed as

$$
\begin{aligned}
& \operatorname{Re} \Delta \mathcal{L}=-\frac{e^{2}}{4 \pi^{3}} a b \sum_{n=1}^{\infty}\left[a_{n}+d_{n}\right] \\
& a_{n}=\frac{\operatorname{coth}(n \pi b / a)}{n}\left\{\mathrm{Ci}\left(\frac{n \pi m^{2}}{e a}\right) \cos \left(\frac{n \pi m^{2}}{e a}\right)+\operatorname{si}\left(\frac{n \pi m^{2}}{e a}\right) \sin \left(\frac{m \pi m^{2}}{e a}\right)\right\} \\
& d_{n}=\frac{-\operatorname{coth}(n \pi a / b)}{2 n}\left\{\exp \left(\frac{n \pi m^{2}}{e b}\right) \operatorname{Ei}\left(-\frac{n \pi m^{2}}{e b}\right)\right. \\
& \left.+\exp \left(-\frac{n \pi m^{2}}{e b}\right) \operatorname{Ei}\left(\frac{m \pi m^{2}}{e b}\right)\right\}
\end{aligned}
$$

while the imaginary part is given as

$$
\operatorname{Im} \Delta \mathcal{L}=\frac{e^{2}|a b|}{8 \pi^{2}} \sum_{n=1}^{\infty} \frac{1}{n} \operatorname{coth}\left(\frac{n \pi a}{b}\right) \exp \left(-\frac{n \pi m^{2}}{e b}\right) .
$$

We note that the Cosine and Sine integrals have an "asymmetric" form since the generally accepted definitions for these integrals are "asymmetric" and are shown below. More details are given in [20].

$$
\begin{gathered}
\operatorname{Ci}(z)=-\int_{z}^{\infty} d t \frac{\cos (t)}{t} \quad z>0 \\
\operatorname{si}(z)=-\int_{z}^{\infty} d t \frac{\sin (t)}{t}=\operatorname{Si}(z)-\frac{\pi}{2} \\
\operatorname{Si}(z)=\int_{0}^{z} d t \frac{\sin (t)}{t} .
\end{gathered}
$$

The imaginary part $\operatorname{Im} \Delta \mathcal{L}$ is generated by a modification of the integration contour in the exponential integral entering into the definition of $d_{n}$ (normally, the exponential integral is defined via a principal-value prescription). The relevant exponential integral is

$$
\begin{aligned}
& \operatorname{Ei}\left(\frac{n \pi m^{2}}{e b}\right) \text { in the definition of } d_{n} \text { and reads } \\
& \operatorname{Ei}(u)=- \text { (P.V.) } \int_{-u}^{\infty} \frac{e^{-t}}{t} d t \quad \text { for } u \in \mathbb{R} .
\end{aligned}
$$


Under an appropriate deformation of the contour, a unified representation for both the real and the imaginary parts is obtained [20],

$$
\begin{aligned}
& \Delta \mathcal{L}=\lim _{\epsilon \rightarrow 0^{+}}-\frac{e^{2}}{4 \pi^{3}} a b \sum_{n=1}^{\infty}\left[b_{n}+c_{n}\right], \\
& b_{n}=-\frac{\operatorname{coth}(n \pi b / a)}{2 n}\left\{\exp \left(-i \frac{n \pi m^{2}}{e a}\right) \Gamma\left(0,-i \frac{n \pi m^{2}}{e a}\right)\right. \\
& \left.+\exp \left(i \frac{n \pi m^{2}}{e a}\right) \Gamma\left(0, i \frac{n \pi m^{2}}{e a}\right)\right\} \text {, } \\
& c_{n}=\frac{\operatorname{coth}(n \pi a / b)}{2 n}\left\{\exp \left(\frac{n \pi m^{2}}{e b}\right) \Gamma\left(0, \frac{n \pi m^{2}}{e b}\right)\right. \\
& \left.+\exp \left(-\frac{n \pi m^{2}}{e b}\right) \Gamma\left(0,-\frac{n \pi m^{2}}{e b}+i \epsilon\right)\right\} \text {. }
\end{aligned}
$$

The effective action has branch cuts along the positive and negative $b$-axis as well as along the positive and negative imaginary axis. For more details, the reader is referred to [20]. In contrast to the exponential integral Ei, the incomplete Gamma function is defined in the entire complex plane with a cut along the negative real axis. It is important to see this connection since the Barnes function 21] is closely connected to the Gamma function which has numerous applications.

The main numerical difficulty is the slow overall convergence of the series expansion whose terms are of nonaltering sign. Padé approximants, a standard tool in many power series application are not capable of summing the series for the HEL 20]. The terms of the convergent series representation are interpreted as being generated by a "partial-fraction decomposition" in close analogy to "partial-wave decomposition" in bound state calculations. It has been shown that the convergence of the HEL series can be accelerated by the same technique as in partial wave decomposition also called the "combined nonlinear-condensation transformation" (CNCT) [20, 22, 23].

Another interesting representation is in terms of the Barnes function. The Barnes function $\mathrm{G}(\mathrm{z})$ is a generalization of the Euler gamma function and is also related to the Hurwitz Zeta function. It obeys the recursion relation [21]

$$
\mathrm{G}(z+1)=\Gamma(z) \mathrm{G}(z) \quad z \in \mathbb{C}, \quad \mathrm{G}(1)=1 .
$$

We write a useful expression for $\log G(z+1)$ below:

$$
\begin{aligned}
\log G(z+1) & =\frac{1}{2} z \log (2 \pi)-\frac{\gamma z^{2}}{2}-\frac{z(z+1)}{2} \\
& +\sum_{k=2}^{\infty}(-1)^{k} \zeta(k) \frac{z^{k+1}}{k+1}, \\
\log \mathrm{G}(z+1) & =z \log (\Gamma(z))+\zeta^{\prime}(-1)-\zeta^{\prime}(-1, z), \\
\zeta^{\prime}(t, z) & =\frac{d}{d t} \zeta(t, z),
\end{aligned}
$$


where $\zeta(k)$ is the Riemann Zeta function, used in the representation of the HEL. Due to its close connection to the $\Gamma(z)$ and the Zeta function, the Barnes function may have a variety of applications in Computer Algebra and Theoretical Physics as well as in other fields.

The Mittag-Leffler theorem (see 20] for a comprehensive discussion) is a key element in the derivation of the series representation for the HEL (some useful formulas, originally derived without the explicit use of the Mittag-Leffler theorem, still based on a partial-fraction decomposition and equivalent to the results obtained by us using the Mittag-Leffler theorem, can be found in [24, p. 271]). The generalization of the Mittag-Leffler theorem for a function with nonsimple poles and zeros is the Riemann-Roch theorem [25, 26]. For Lagrangians with poles and zeros of higher order, which can occur in string theories, we anticipate the application of the Riemann-Roch theorem. Such Lagrangians with poles and zeros of arbitrary order may form a vector space of meromorphic functions over a complex field.

\section{MAGNETO-OPTICAL EFFECT: SECOND AND HIGHER-HARMONIC GENERATION}

Maxwell's equations receive corrections from virtual excitations of the charged quantum fields(notably electrons and positrons). This leads to interesting effects [1]: light-by-light scattering, photon splitting, modification of the speed of light in the presence of strong electromagnetic fields, and - last, but not least - pair production. The dominant effect for electromagnetic fields that vary slowly with respect to the Compton wavelength (frequencies $\omega \ll 2 m c^{2} / h$ ) is described by the Heisenberg-Euler Lagrangian, which is known to all orders in the electromagnetic field. For the case of zero electric field, the HEL can be written as [18, 19]

$$
\Delta \mathcal{L}(h)=\frac{m^{4}}{32 \pi^{2}}\left[\left(\frac{1}{h^{2}}\right)\left\{-\left(\frac{1}{3}+2 h+2 h^{2}\right) \ln h+h^{2}-4 L_{1}+4 \ln \Gamma_{1}(1+h)\right\}\right],
$$

where $h=\frac{H_{c r}}{2 H}=\frac{m^{2}}{2 e H}, L_{1} \cong 0.2487, H_{c r} \cong 4.4 \times 10^{13}$ Gauss and $\Gamma_{1}(h)$ is the generalized $\Gamma$ function. We observe that the expression in the braces for the HEL contains terms with quadratic powers of $h$ as well as terms like $h \ln h$ and $h^{2} \ln h$. It is well known that the logarithm of a trigonometric Cosine function has even powers of the argument when expressed as a power series [16, 27, 28. This is the situation encountered for $\Delta \mathcal{L}(h)$ when the magnetic field $\mathrm{H}$ (also meant as B) is expressed as a cosine wave for a plane electromagnetic wave propagation [16]. The cases of parallel and perpendicular polarizations for the weak and strong field cases and the evaluation of the higher harmonics have been treated in detail earlier in [16]. Equation (28) describes the case for arbitrary field strengths and generalizes the evaluation for the generation of the higher harmonics.

Magneto-optical Effect We briefly discuss second and higher-harmonic generation in a static magnetic field which is an example of broken symmetry. By broken symmetry, we mean a violation of the principle of superposition/linearity. High intensity synchrotron radiation, storage rings and lasers are good experimental tools for the study of higher-harmonic generation. The degeneracy of nonlinearity is broken by a static field or nonplanar or nonmonochromatic waves and leads to the generation of higher harmonics. We define,

$$
x=\frac{1}{\pi F_{c}}\left[-S+\left(S^{2}+P^{2}\right)^{\frac{1}{2}}\right]^{\frac{1}{2}}, \quad y=\frac{1}{\pi F_{c}}\left[S+\left(S^{2}+P^{2}\right)^{\frac{1}{2}}\right]^{\frac{1}{2}},
$$


in analogy to a and b of equations (5) and (6) above. Here $F_{c}=H_{c r}$. For

$$
\begin{aligned}
& x=0, \quad y \neq 0, \quad|P|=0, \quad \text { we obtain } \\
& \operatorname{Re} \mathcal{L}_{\text {eff }}=S-\frac{\alpha}{\pi} F_{c}^{2} \sum_{k=1} \tilde{d}_{k},
\end{aligned}
$$

where,

$$
\tilde{d}_{k}=-\frac{y^{2}}{2 k^{2}}\left[e^{\frac{k}{y}} \operatorname{Ei}\left(\frac{-k}{y}\right)+e^{-\frac{k}{y}} \operatorname{Ei}\left(\frac{k}{y}\right)\right]=-\frac{y^{2}}{k^{2}} \sum_{N=1}^{\infty}(2 N-1) !\left(\frac{y}{k}\right)^{2 N} .
$$

Again, it is to be noted that Ei in Equation (31) is defined as real for real (negative and/or positive) arguments.

We use the formulas: $\zeta(4)=\frac{\pi^{4}}{90}, \zeta(6)=\frac{\pi^{6}}{945}$ etc. to derive the equations above and below. $\tilde{d}_{k}$ can be evaluated in the case of an external static magnetic field $B_{0}$ along the x-axis. For an electromagnetic wave propagating along the $\mathrm{z}$ axis, we have for the case [7],

$$
\begin{aligned}
E_{x} & =E_{z}=0, \quad B_{y}=B_{z}=0, \quad\left|E_{y}\right|=\left|B_{x}\right|, \quad P=E \cdot B=0, \\
S & =\frac{1}{2}\left[E_{y}^{2}-\left(B_{x}+B_{0}\right)^{2}\right]=-\frac{1}{2}\left(B_{0}^{2}+2 B_{x} B_{0}\right) .
\end{aligned}
$$

We then derive an expression for the partial derivative of the $\operatorname{Re} \mathcal{L}_{\text {eff }}$ with respect to $\mathrm{S}$ :

$$
\frac{\partial}{\partial S} \operatorname{Re} \mathcal{L}_{\text {eff }}=1+\frac{\alpha}{\pi}\left[\frac{4}{4 S} \frac{S}{F_{c}^{2}}+\frac{16}{105} \frac{S^{2}}{F_{c}^{4}}+\frac{256}{315} \frac{S^{3}}{F_{c}^{6}}+\ldots\right]
$$

The electric displacement field D can then be obtained.

$$
D=\frac{\partial}{\partial S} \operatorname{Re} \mathcal{L}_{e f f} \cdot E
$$

We also observe that

$$
\frac{\partial P}{\partial E} \cdot \frac{\partial}{\partial P} \operatorname{Re} \mathcal{L}_{e f f}=0
$$

We consider electromagnetic waves with $E_{y}$ and $B_{x}$ of the form:

$$
\begin{aligned}
& E_{y}=A \cos \phi=A \cos (w t-k z), \\
& B_{x}=A \cos \phi=A \cos (w t-k z) .
\end{aligned}
$$

We then find that,

$$
D_{y} \cong E_{y}\left[1+\frac{4 \alpha}{45 \pi}\left\{-\left(\frac{B_{0}^{2}+2 B_{x} B_{0}}{2 F_{c}^{2}}\right)+\frac{3}{7}\left(\frac{B_{0}^{2}+2 B_{x} B_{0}}{F_{c}^{2}}\right)^{2}\right\}\right],
$$

where $A$ is the amplitude of the wave. We find that the parallel mode propagates alone. The $E_{y} B_{x}$ terms in equation (39) indicate the presence of the second harmonic, as can be observed from the fact that:

$$
2 E_{y} B_{x}=2 A^{2} \cos ^{2} \phi=A^{2}(1+\cos 2 \phi) .
$$


The term proportional to $\cos (2 \phi)$ indicates the presence of the second harmonic. Similarly, $E_{y} B_{x}^{2}$ indicates the presence of the third harmonic and $E_{y} B_{x}^{3}$ indicates the fourth harmonic etc. In a similar way, the evaluation of $H$ can be done:

$$
H=-\frac{\partial S}{\partial B} \cdot \frac{\partial}{\partial S} \operatorname{Re} \mathcal{L}_{e f f}(P=0) .
$$

The above expression of $H$ is also shown to exhibit the second and higher harmonics. The spatial anisotropy is imposed by the magnetic field. The SHG depends on propagation direction and polarization direction of the fundamental electromagnetic wave with respect to $B_{0}$. The maximum effect is when $\hat{B}_{0}$ is $\perp \hat{k}$ (the wave propagation direction).

\section{CONCLUSIONS}

We have investigated questions related to the representation of the quantum electrodynamic (QED) effective Lagrangian and its analytical expression. In Sect. 2, we briefly recalled our previous results given for special-function representations of the effective Lagrangian, and we briefly clarify the mathematical notation used in the special-function representations (12) and (21). The representation (21) unifies the real and imaginary parts. We also introduced the Barnes function that should be of use in a variety of applications that include the HEL. We very briefly mention that the key step in the derivation of our series representations is the Mittag-Leffler theorem [20].

In section 3, we have also given an expression from the effective Lagrangian that will facilitate the evaluation of the higher harmonics. We also briefly discuss the magneto-optical effect of the vacuum which might provide a signature of "QED's nonlinear light." In the Appendix, we discuss some aspects of the renormalization and the renorm-invariance of the action. Based on the results of the current paper, we expect to carry out detailed studies related to various projected and ongoing experiments and astrophysical phenomena [1,11-17] involving strong static-field conditions (or fields with frequencies that are small as compared to the electron Compton wavelength). The study of the HEL to include finite temperature effects [29] with the series representation that we have developed is an interesting problem that warrants further investigation.

\section{ACKNOWLEDGEMENTS}

The authors wish to acknowledge insightful conversations with Dr. Holger Gies and Professors Bowick and Joe Schecter of the Physics Department, Syracuse University. They would also like to thank Kato Lo and John Drozd for helpful discussions and invaluable assistance in processing the paper. S.R.V. acknowledges a supporting grant from the Natural Sciences and Engineering Research Council of Canada (NSERC).

\section{A APPENDIX - RENORMALIZATION AND RENORMALIZATION-GROUP INVARIANCE}

The renormalization as well as the renormalization-group invariance of the QED effective Lagrangian have already been discussed at length by various authors (see [30] and references therein). 
However, material on this topic is somewhat scattered in the literature. In the current Appendix, we would like to give a brief overview of the physical ideas that led to the formulation of the related RG equations, as well as the connection with the $\beta$ functions of QED, complemented by an easy-to-understand presentation of the basic notion of the renormalization itself.

It should be noted that the considerations in this section have only partial significance for the main topic of the current investigation, which is a treatment of higher-harmonic generation based on series representations of the Lagrangian. The current section merely provides illustrating remarks on the derivation of the effective Lagrangian, which is again the starting point of the main endeavour pursued.

The "original" Lagrangian of QED from which we start to work out the $S$-matrix with all its radiative corrections in fact has to be identified with the "bare" Lagrangian that is expressed in terms of the "bare" physical quantities (bare charges, masses, fields). As we develop the perturbations series (in $e^{2}$ ), we see that some terms (it does not matter if they are infinite or not) in fact modify the physical parameters that entered into the very Lagrangian from which our work started. This means that we have to renormalize the Lagrangian.

Renormalization: The renormalization can be done by adding counterterms to the Lagrangian. These terms relate the bare parameters (bare charges, masses, fields) to the physical parameters of the theory, i.e. to the renormalized charges, masses, fields. The renormalizability then requires that the Renormalized Lagrangian $=$ Bare Lagrangian + Counterterms $=$ Bare Lagrangian but with charges, masses, fields expressed in terms of the renormalized parameters. For QED, we are in the lucky position that the theory remains finite in the infrared, i.e. that the physical charge of the electron remains finite as we move two electrons far apart. Therefore, we may renormalize QED on mass shell, i.e. renormalize QED in such a way that the renormalized charge is by definition the charge of an electron as seen by another electron in the limit of a large separation of the two electrons (this latter situation corresponds to the limit of a very soft exchanged photon - the infrared limit in which the wave vector $k$ of the exchange photon tends to zero).

To see how this works, look at p. 325 of [31]: The vacuum polarization modifies the Coulomb law (or Thompson scattering) in such a way that ( $e_{0}$ is the bare charge)

$$
e_{0}^{2} / k^{2} \rightarrow e_{0}^{2} /\left[k^{2}\left(1+\omega\left(k^{2}\right)\right)\right]=e_{0}^{2} /\left[k^{2}\left(1+\omega(0)+O\left(k^{2}\right)\right)\right] .
$$

Now at $k=0$, the physical charge of the electron is obtained, which means that $e^{2}=e_{0}^{2} /(1+$ $\omega(0)$ ), or that the $Z_{3}$ renormalization that relates the bare and the renormalized charge of the electron is obtained as $Z_{3}=1 /(1+\omega(0))$ where $e^{2}=Z_{3} e_{0}^{2}$. This relates the physical renormalized charge with the bare charge of the electron. In order to construct a renormalized Lagrangian in which the " $e$ " is indeed the renormalized, finite electron charge, we now have fulfill the condition that the vacuum polarization as derived from our renormalized Lagrangian results in a "modified" vacuum polarization correction to the Coulomb law that fulfills $\omega_{\text {Renormalized }}(0)=0$. This is just Eq. (8-96e) of [31]. In general, a set of renormalization conditions imposed on the renormalized Lagrangian determines the physical interpretation of the parameters in terms of which the renormalized Lagrangian is written. The renormalization conditions (8-96) in [31, on which the considerations of [20] are based, ensure that the renormalized effective Lagrangian is written in terms of the finite, renormalized, physical electron charge. The "usual" representation of the QED Effective Lagrangian (QED Effective Action) in Eq. (1) fulfills these conditions.

In other words, since we are used to defining the value of the coupling by Thompson scattering, i.e., by scattering a long-wavelength $(\omega \rightarrow 0)$ photon off a (static) electron, the renormalization scale is naturally set by the mass of the electron. This amounts to the so-called on-electron- 
mass - shell renormalization condition $e^{2}(\mu=m) /(4 \pi) \simeq 1 / 137.036$. Our starting point Eq. (1) for the HEL is obtained exactly by implementing this renormalization condition, and the coupling $e$ used throughout this work should be interpreted in this way. It is precisely the second term in square brackets in Eq. (1) that guarantees this renormalization condition. This term subtracts any contribution of $\Delta \mathcal{L}$ to Thompson scattering; therefore, the latter is fully described only by the renormalized Maxwell term together with the static-eletron interaction term involving the on-shell renormalized coupling $e$ - as it should. This renormalization procedure has thereby fixed all free parameters, such that the theory is now fully predictive for all other processes that can be described by our one-loop HEL.

QED is usually defined at some high ultraviolet (UV) scale in terms of a bare action. It is predictive for electrodynamic processes at low energy scales to a high accuracy, once all renormalization-group "relevant" parameters are specified. QED has two such parameters, the coupling $e$ and the electron mass $m$. During the derivation of the HEL (1), we have to give a prescription how to fix these parameters to their physical values, since they turn out to be scaledependent. However, since the HEL of Eq. (1) describes processes with external photon lines to one loop only, the scale dependence of $m$ remains invisible in this calculation. Hence, to this order of accuracy, we can fix the electron mass to its value measurable at the scale of, say, atomic physics, and insert this into Eq. (1). In the HEL language, the scale dependence of the mass becomes visible at two-loop order, see, e.g., 30,32,33. This subtlety emerges in the following way: The unrenormalized two-loop expression contains a term which may be written as $\delta m^{2}\left(\partial / \partial m^{2}\right)$ (one-loop Lagrangian), where $\mathrm{m}$ is the electron mass and $\delta m$ the radiative modification (the physical mass is then $m_{\mathrm{ph}}^{2}=m^{2}+\delta m^{2}$. The original Maxwell Lagrangian is independent of the electron mass. The one-loop Lagrangian that we work with depends on the electron mass. The two-loop Lagrangian has effective self-energy corrections to the dressed fermion propagators that interact with themselves through a further radiative photon. This self-energy effect is known to produce a radiative renormalization $\delta m$ of the electron mass. Therefore, the above term has to be interpreted as a correction to the electron mass that enters into the one-loop Lagrangian, expressing the fact that "one-loop Lagrangian $+\delta m^{2}\left(\partial / \partial m^{2}\right)$ (one-loop Lagrangian) = one-loop Lagrangian as a function of $m_{\text {Renormalized }}^{2}=m^{2}+\delta m^{2}$." This is the mechanism by which the anomalous mass dimension creeps into the renormalization group analysis of the action [30].

Renormalization-Group Invariance: The QED Effective action is well known to be a modification of the Maxwell Lagrangian relevant to the situation of strong background fields. By contrast, vacuum polarization modifies the Coulomb at small distances (large momenta). However, there is a connection between the two regimes, asymptotically. Specifically, Ritus (see 32 and references therein) has shown that an interesting connection exists between the corrections to the Maxwell equations in intense fields and vacuum-polarization corrections to QED at large momenta. The connection can now be found upon considering Eqs. (25) and (26) of Ref. [32]. Observe that the mass renormalization in Eq. (14) of Ritus also has the right asymptotic behaviour at small proper time $s_{0}$. One of the basic ideas of the renormalization group invariance is that the product of field and charge, which enters into the covariant coupling, $\mathrm{i} \partial_{\mu}-e_{0} A_{\mu}^{0} \rightarrow \mathrm{i} \partial_{\mu}-e A_{\mu}$ in going from bare to renormalized quantities, necessarily has to be preserved under the renormalization because space itself $\left(\partial_{\mu} \equiv \partial / \partial x_{\mu}\right)$ does not stretch under the renormalization of charge and field. The charge and the field are renormalized as $e_{0}=Z_{3}^{-1 / 2} e$ and $A_{\mu}^{0}=Z_{3}^{1 / 2} A_{\mu}$. [Here, "0" denotes the bare quantity.]

Let us finally discuss the scale dependence of the coupling $e$ as it occurs in the HEL. Starting the computation with a bare (Maxwell) action at UV scale $\Lambda$ with a bare coupling $e_{0} \equiv e_{0}(\Lambda)$, we obtain a $\Lambda$ - and $e_{0}$-dependent contribution to the Maxwell action $\sim \mathcal{F}$. Together with the bare 
action, we can trade these contributions for a scale-dependent coupling $e=e(\mu)$ that describes the interaction of a photon with an electron at an energy scale $\mu$ [5, 30, 32. Of course, $\mu$ is an arbitrary parameter, and the requirement that no physical process should depend on $\mu$ defines how the value of the coupling parameter $e(\mu)$ has to be changed upon a variation of $\mu$. For quantitative predictivity, we have to fix the value of $e(\mu)$ at one particular scale $\mu$. Starting from this consideration, one may derive functional relationships for the asymptotic behaviour of the QED Effective Action in intense fields, which in turn enable the direct transition to renormalization-group equations of the Callan-Symanzik type [see Eq. (45) of [32]]. Other types of renormalization-group equations, for example those of the Gell-Mann Low type, including a thorough discussion, can be found in [5] and [30].

Of course, the dependence on $\mu$ of the HEL could be formally kept such as in [10] which would correspond to not specifying a renormalization condition. However, once the input of a single experiment (e.g., Thompson scattering) is taken into account, no freedom is left in the parameters, the $\mu$-dependence has disappeared and QED is fully predictive. In particular, there is no room for further "logarithmic correction terms" [10].

\section{References}

[1] W. Dittrich and H. Gies. Probing the quantum vacuum. Springer Tracts in Physics, Vol. 166 (Springer, Berlin, 2000).

[2] W. Heisenberg and H. Euler. Z. Phys. 98, 714 (1936).

[3] V. Weisskopf. K. Dan. Vidensk. Selsk. Mat. Fys. Medd. 14, 1 (1936). Reprinted on pp. 92-128 of J. Schwinger (Editor). Selected papers on quantum electrodynamics (Dover, New York, 1958).

[4] J. Schwinger. Phys. Rev. 82, 664 (1951). Reprinted on pp. 209-224 of J. Schwinger (Editor). Selected papers on quantum electrodynamics. Dover, New York. (1958).

[5] W. Dittrich and M. Reuter. Effective Lagrangians in quantum electrodynamics. Lecture notes in physics vol. 220 (Springer, Berlin, 1985).

[6] Z. Bialynicka-Birula and I. Bialynicki-Birula. Phys. Rev. D: Part. Fields 2, 2341 (1970).

[7] Z. Bialynicka-Birula. Acta. Phys. Pol. A57, 729 (1980).

[8] S.R. Valluri, D. Lamm, and W.J. Mielniczuk. Can. J. Phys. 71, 389 (1993).

[9] S.R. Valluri, D. Lamm, and W.J. Mielniczuk. Can. J. Phys. 72, 786 (1994).

[10] Y.M. Cho and D.G. Pak. Phys. Rev. Lett. 86, 1947 (2001); Y.M. Cho and D.G. Pak. Phys. Rev. Lett. 91, 039101 (2003).

[11] S.L. Adler. Ann. Phys. 67, 599 (1971).

[12] A. Salam and J. Strathdee. Nucl. Phys. B90, 203 (1975).

[13] R.J. Stoneham. J. Phys. A: Math. Gen. 12, 2187 (1979).

[14] R.C. Duncan and C. Thompson. Astrophys. J. 392, L9 (1992). 
[15] M.G. Baring. Astrophys. J. 440, L69 (1995).

[16] S.R. Valluri and P. Bhartia. Can. J. Phys. 58, 116 (1980) and Can. J. Phys. 56, 1122 (1978).

[17] J.S. Heyl and L. Hernquist. J. Phys. A: Math. Gen. 30, 6485 (1997) and Phys. Rev. D: Part. Fields 55, 2449 (1997).

[18] S.R. Valluri, D. Lamm, and W.J. Mielniczuk. Phys. Rev. D: Part. Fields 25, 2729 (1982). See also: L. Bendersky, Acta Math. 61, 263 (1933).

[19] W. Dittrich, W.Y. Tsai, and K.H. Zimmermann. Phys. Rev. D: Part. Fields 19, 2929 (1979).

[20] D. R. Lamm, S. R. Valluri, U. D. Jentschura, and E. J. Weniger. Phys. Rev. Lett. 88, 089101 (2002); D. R. Lamm, S. R. Valluri, H. Gies, U. D. Jentschura, and E. J. Weniger. Can. J. Phys. 80, 267 (2002).

[21] V. Adamchik. Applied Mathematics and Computation, 134, 515 (2003); Proceedings of the 2001 International Symposium on Symbolic and Algebraic Computation, July 22-25, 2001, London, Canada, (Academic, New York, 2001), pp.15-20.

[22] E.J. Weniger. Nonlinear sequence transformations: e-print math.CA/0107080 (2001).

[23] E.J. Weniger. Comput. Phys. Rep. 10, 189 (1989).

[24] B.C. Berndt. Ramanujan's notebooks. Part II. Springer, Berlin. 1989.

[25] J. Dieudonne. History of algebraic geometry (Wadsworth, Monterey, 1985).

[26] P. Griffith and J. Harris. Principles of algebraic geometry (John Wiley and Sons, New York, 1978).

[27] I. Gradshtein and I. Ryzhik. Tables of series, integrals, and products (Harry Deutsch, Thun and Frankfurt am Main, 1981).

[28] M. Abramowitz and I.A. Stegun. Handbook of mathematical functions. 10th ed. (National Bureau of Standards, Washington, D.C., 1972).

[29] W. Dittrich, Phys. Rev. D 19, 2385 (1979)

[30] G. V. Dunne, H. Gies and C. Schubert, JHEP 11, 032 (2002).

[31] C. Itzykson and J. B. Zuber, Quantum Field Theory (McGraw-Hill, New York, 1980).

[32] V. I. Ritus, Effective Lagrange function of an intense electromagnetic field in QED, Proceedings of the conference "Frontier Tests of QED" (Sandansky, Bulgaria, 9-15 June, 1998); Eds. E. Zavattini, D. Bakalov, C. Rizzo (Heron Press, Sofia, 1998); e-print hep-th/9812124.

[33] D. Fliegner, M. Reuter, M. G. Schmidt and C. Schubert, Teor. Mat. Fiz. 113N2, 289 (1997) [Theor. Math. Phys. 113, 1442 (1997)]; e-print hep-th/9704194. 